\title{
PROGRAMAÇÃO DA PRODUÇÃO EM FLOW SHOP PERMUTACIONAL ENVOLVENDO MEDIDAS DE ATRASO: UMA CONTRIBUIÇÃO BIBLIOMÉTRICA A PARTIR DA BASE DE DADOS WEB OF SCIENCE
}

\author{
P. A. Hordones ${ }^{*}$, H. Fuchigami \\ Universidade Federal de Goiás \\ ecn.pauloantonio@gmail.com *
}

Submetido 02/03/2017 - Aceito 28/11/2017

DOI: $10.15628 /$ holos.2017.5711

\section{RESUMO}

A organização de dados sobre a produção científica de um período contribui para a continuidade da pesquisa e para o desenvolvimento de um campo particular do conhecimento. O principal objetivo desta pesquisa é conhecer o panorama da produção científica sobre programação da produção em sistemas flow fhop permutacional envolvendo medidas de atraso, de maneira quantitativa, identificando os periódicos de destaque, autores que mais abordaram o tema e os trabalhos mais representativos. Tratou-se de um estudo de análise bibliométrica, através da base de dados Web of Science e seu tratamento pelos softwares
ResearcherID ${ }^{\circledR}$ e VosViewer ${ }^{\circledast}$. Para a pesquisa, utilizouse os seguintes expressões-chave: "permu ${ }^{*}$ flow ${ }^{*} e$ tard *" ou "permu * flow* e atras*", gerando uma amostra constituída por 133 artigos. Ao final do estudo, apresentou-se resultados de estatística descritiva com várias identificações, como os principais autores e os artigos e periódicos mais referenciados por estudiosos em todo o mundo. Além das principais redes sociais: de citações e cocitações. Tem-se neste trabalho, portanto, um importante aliado no desenvolvimento de novas abordagens sobre 0 tema.

PALAVRAS-CHAVE: programação da produção, sequenciamento da produção, análise de redes, análise bibliométrica.

\section{PERMUTATION FLOW SHOP SCHEDULING PROBLEM INVOLVING TARDINESS MEASUREMENTS: A BIBLIOMETRIC CONTRIBUTION FROM THE WEB OF SCIENCE DATABASE}

\begin{abstract}
The organization of data on the scientific production of a period contributes to the continuity of research and development of a particular field of knowledge. The main objective of this study is therefore to quantitatively analyze what occurred in relation to the programming problem in Production Systems Flow shop permutation, involving tardiness measurements. This was a study of bibliometric analysis, using data from the database Web of Science and its treatment by ResearcherID ${ }^{\circledR}$ and VosViewer ${ }^{\circledR}$ software. For the research, we used the following key terms: "permu*
\end{abstract}

KEYWORDS: production planning, scheduling, network analysis, bibliometric analysis. 


\section{INTRODUÇÃO}

A globalização da economia mundial e a generalização da internet mudaram a estratégia de produção de muitos setores industriais. Várias indústrias passaram a atender um grande número de clientes espalhados por todo o mundo (M'Hallah, 2014).

Para se manterem competitivas e oferecer uma vantagem sustentável, muitas pequenas e médias empresas passaram a planejar sua produção baseadas na quantidade de pedidos que recebem diariamente. A vantagem competitiva dessas empresas é atender a data de entrega que cada cliente necessita, independentemente do tamanho da ordem ou do destino da mercadoria (Schaller, 2012).

Neste contexto extremamente competitivo em que as empresas disputam cada vez mais clientes, reconhecimento e condições de permanecer competitivas e lucrativas, é fundamental o desenvolvimento de mecanismos que as auxiliem na tomada de decisões.

Para Lopez \& Roubellat (2013), o investimento em programação da produção (scheduling) pelas empresas tornou-se uma necessidade de sobrevivência. As empresas devem programar atividades de forma a utilizar os recursos disponíveis de maneira eficiente, a fim de minimizar custos e respeitar compromissos de entrega estabelecidos com os clientes.

De acordo com Endler et al. (2015) a programação da produção tem sido objeto de estudo altamente explorado pelas comunidades acadêmicas nas últimas décadas. Para os autores, o avanço científico nesse campo é fruto de investigações e interações entre os pesquisadores ao longo do tempo.

Os problemas de programação de tarefas em sistemas de produção são, tradicionalmente, classificados em função do fluxo das operações nas máquinas: máquina única, máquinas paralelas, flow shop, flow shop permutacional, job shop, open shop, flow shop com máquinas múltiplas, job shop com máquinas múltiplas.

O presente trabalho trata, especificamente, do problema de programação de operações em ambiente flow shop permutacional (FSP), definido por Fuchigami \& Rangel (2015) como um sistema produtivo em que as tarefas seguem o mesmo fluxo por todas as máquinas na mesma ordem. De acordo com os autores, é um dos mais importantes problemas de planejamento da produção. Pode ser encontrado em ambientes industriais de produção intermitente, como: indústrias químicas, eletrônicas, fundições, processamento de fibra de vidro dentre outras (Kim, 1995; Tasgetiren et al., 2013).

O objetivo da pesquisa foi conhecer o panorama da produção científica sobre programação de operações em sistemas de produção FSP envolvendo medidas de atraso de maneira quantitativa, identificando os periódicos de destaque, autores que mais abordaram o tema e os trabalhos mais representativos. A abordagem metodológica utilizada foi a de revisão sistemática da literatura, tendo como base a teoria bibliométrica e a análise de redes.

O trabalho está estruturado em cinco etapas, contadas com essa introdução. Nas próximas seções será apresentada a revisão bibliográfica, expondo os principais conceitos e trabalhos encontrados que abordaram especificamente o objeto desta pesquisa, a descrição do 
método de seleção da amostra de artigos e a análise dos resultados encontrados. Na última seção serão apresentadas as considerações finais.

\section{REVISÃO BIBLIOGRÁFICA}

A programação da produção ou scheduling surgiu como uma área de pesquisa na década de 1950 com a publicação do trabalho de Johnson (1954). Desde então, vem recebendo considerável atenção de estudiosos da pesquisa operacional, matemáticos e pesquisadores da área de produção e operações. Nos últimos 60 anos uma significativa quantidade de obras que tratam dos problemas de programação foi formulada utilizando diversas configurações de máquinas, diferentes restrições e variadas funções objetivo.

De acordo com Baker (1974), o problema de programação da produção em geral, requer decisões de sequenciamento e alocação de recursos. Tais recursos podem ser ferramentas, materiais, máquinas etc. Para Pinedo (2012), scheduling é um problema de priorização de atividades que incluem a alocação de recursos limitados, ao longo do tempo, para realização de tarefas, de modo a satisfazer os objetivos estabelecidos.

Os problemas de programação da produção podem ser classificados de acordo com a nomenclatura composta por três campos $(\alpha / \beta / \gamma)$ proposta por Graham et al. (1979). Essa nomenclatura possibilita a representação do ambiente de produção com o número de máquinas ou estágios $(\alpha)$, detalhes das propriedades e limitações dos recursos e tarefas $(\beta)$ e a função objetivo (medida de desempenho) a ser minimizada/maximizada $(\gamma)$, de maneira compacta.

Fuchigami (2015) categoriza o ambiente de produção $(\alpha)$ em função do fluxo das operações nas máquinas: máquina única: máquinas paralelas, flow shop, flow shop permutacional, job shop, open shop, flow shop com máquinas múltiplas, job shop com máquinas múltiplas.

Exemplos de possíveis dados de entrada no campo " $\beta$ " são: data de liberação ( $r j)$, prazos de entrega (dj), tempos de setup dependentes da sequência (sij), tempos de setup antecipado (as), tempos de setup não antecipados (ns), operações podem ser interrompidas (prmp), a solução deve ser permutacional (prmu), as máquinas podem estar continuamente disponíveis (brkdwn), as operações podem ser subdivididas (split), capacidade finita de buffer entre duas máquinas (block), tarefas não podem estar entre duas máquinas sucessivas (nwt), tarefas podem visitar uma máquina mais de uma vez (recrc) etc.

As medidas mais usuais presentes no campo $(\gamma)$, denominadas medidas de desempenho regulares, indicadas por Fuchigami (2015) são: duração total da programação (Cmax), tempo médio de fluxo (F), maior diferença da pontualidade das tarefas (Lmax), maior atraso das tarefas (Tmax), maior adiantamento das tarefas (Emax), soma das datas de término das tarefas $\left(\sum \mathrm{Cj}\right)$, número de tarefas em atraso $(\mathrm{nT})$, total de tempo ocioso das máquinas $\left(\sum \mathrm{lm}\right)$, soma ponderada das datas de término das tarefas $\left(\sum\right.$ wj $\left.\mathrm{Cj}\right)$, soma ponderada do atraso das tarefas $\left(\sum \mathrm{wj} \mathrm{Tj}\right)$, etc.

\subsection{O Problema de programação flow shop permutacional e as medidas de atraso}

O ambiente de produção flow shop permutacional é ilustrado na Figura 1.

De acordo com Moccelin \& Nagano (2007), o problema consiste em programar um conjunto de $n$ tarefas, definido como $J=\{J 1, \ldots, J n\}$, onde se mantém a mesma ordem de 
programação destas $n$ tarefas em todas as máquinas. O objetivo é encontrar uma sequência, entre as $n$ ! possíveis, para o processamento das tarefas nas máquinas, de modo que alguma medida de desempenho previamente estabelecida seja otimizada.

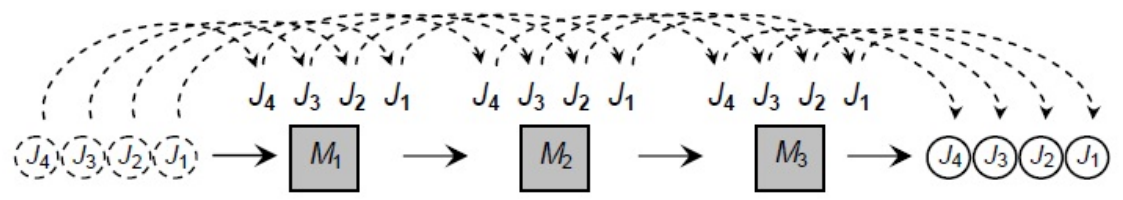

Figura 1: Ilustração de um flow shop permutacional

Fonte: Elaborada pelos autores

Na literatura científica, conforme constatou Fuchigami \& Rangel (2015), as medidas de desempenho comumente encontradas referem-se: à minimização do tempo médio de fluxo (mean flow time), associado à redução do estoque em processamento; à minimização da duração total da programação (makespan), associada à utilização eficiente dos recursos produtivos; ou então as derivadas do atraso, consideradas neste estudo: atraso total, atraso máximo, número de tarefas atrasadas e atraso ponderado.

\subsection{Métodos de Solução do Problema}

Maccarthy \& Liu (1993) categorizam os métodos de solução para problemas de programação da produção em: (i) métodos ótimos (ou exatos): geram a melhor programação possível de acordo com o critério de desempenho adotado, e (ii) métodos heurísticos: geram uma programação de boa qualidade sem garantia de otimalidade em um tempo computacional aceitável.

Os métodos heurísticos podem ser classificados como construtivos ou melhorativos, conforme descrito em Moccellin (1999). No caso dos métodos construtivos, a sequência adotada como solução do problema é obtida: (a) diretamente a partir da ordenação das tarefas segundo índices de prioridade calculados em função dos tempos de processamento das tarefas, como Palmer (1965) e Gupta (1971); (b) escolhendo-se a melhor sequência das tarefas a partir de um conjunto de sequências também obtidas utilizando-se índices de prioridade associados às tarefas, como Campbell et al. (1970) e Hundal \& Rajgopal (1988); (c) a partir da geração sucessiva de sequências parciais das tarefas, até a obtenção de uma sequência completa através de algum critério de inserção de tarefas, como Nawaz et al. (1983) e Fernandez Viagas \& Framiam (2015).

Um tipo de heurística bastante estudada nas últimas décadas são as meta-heurísticas, definidas por Osman \& Laporte (1996), como um processo interativo responsável por guiar uma heurística subordinada, combinando de forma inteligente diferentes conceitos a fim de explorar o espaço de busca. Um aprofundamento acerca das meta-heurísticas utilizadas para resolver problemas com medidas derivadas do atraso em flow shop permutacional podem ser encontrados em: Framinam \& Leisten (2008), Madhushini et al. (2009), Vallada \& Ruiz (2010), Schaller (2012), Xiao et al. (2012), Schaller \& Valente (2013), Tasgetiren et al. (2013), Lee et al. (2014) e Fernandez Viegas \& Framinam (2015).

\section{METODOLOGIA}

Este estudo foi desenvolvido a partir de uma pesquisa bibliométrica, objetivando ampliar o conhecimento referente às publicações relacionadas à programação da produção em flow shop permutacional envolvendo medidas de atraso. 
A bibliometria é definida por Pilkington \& Meredith (2009) como a técnica de investigação que objetiva a análise do tamanho, crescimento e distribuição bibliográfica num determinado campo do conhecimento. Segundo Gumpenberger \& Gorraiz (2012), a bibliometria é uma disciplina das ciências da biblioteca e da informação, sendo desenvolvida como uma ferramenta para medir e monitorar a produção científica. Para Silva (2004), a bibliometria objetiva analisar a atividade cientifica ou técnica por meio do estudo quantitativo das publicações. Em recente trabalho, Alves et al. (2016), enfatizam a importância dos indicadores de desempenho bibliométricos para avaliar a pesquisa acadêmica e orientar rumos e estratégias de financiamento de pesquisas.

\subsection{Definição da amostra}

A amostra de artigos sobre o problema de programação flow shop permutacional envolvendo medidas de atraso foi definida a partir da escolha da base de dados, da identificação das palavras-chave, do tipo e dos anos das publicações, conforme ilustrado no fluxograma da Figura 2.

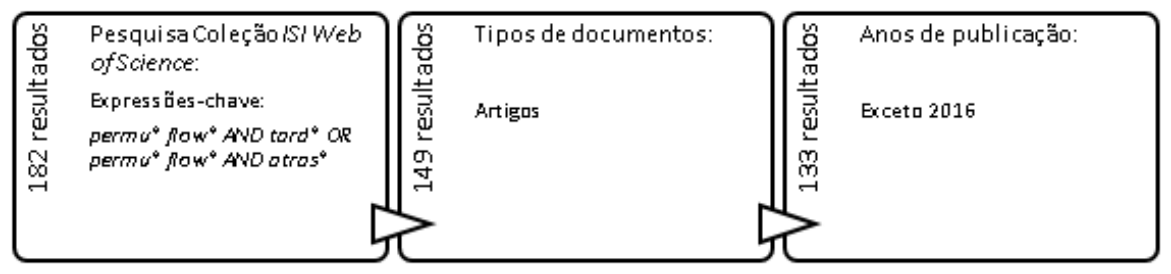

Figura 2: Fluxograma do processo de definição da amostra

Fonte: Elaborada pelos autores

Quanto à determinação da base de dados, a opção pela utilização da Web of Science (WoS) como principal fonte de informação da pesquisa leva em conta critérios de tradição, credibilidade, visibilidade (Packer e Meneghini, 2006), pela fato desta base incluir revistas com fator de impacto calculado no Journal Citation Report (JCR), por sua característica multidisciplinar e sua abrangência (Testa, 2011). Considerou-se também, o fato da WoS ser uma das fontes de dados mais empregadas em estudos métricos e as vantagens que oferece em relação às demais bases (Carvalho et al., 2013). Soma-se, o fato da WoS ser uma base de dados multidisciplinar, integrada à base ISI (Institute for Scientific Information) Web of Knowledge, que indexa publicações científicas de mais de 12.000 periódicos (Wos, 2016).

No que se refere à determinação do tipo de publicação - livros, artigos, anais de congresso, etc. - optou-se por considerar apenas artigos. A escolha é válida pois, artigos acadêmicos geralmente antecedem livros consagrados e são considerados fontes seguras para pesquisas por apresentarem rigor metodológico para publicação. Excluiu-se o ano de 2016, pelo fato de se desejar realizar uma busca em um ano completo, com 12 meses. Inicialmente a pesquisa retornou um total de 182 artigos, que após o refinamento resultou em uma amostra de 133 artigos a serem analisados.

\subsection{Organização e tratamento bibliométrico da amostra}

O tratamento bibliométrico dos registros coletados é apresentado em duas etapas: estatística descritiva e análise de redes sociais. A análise de redes sociais é uma técnica 
interdisciplinar, desenvolvida pela matemática e ciência da computação. Na bibliometria vem se consolidando como ferramenta para o estudo de interações e relações sociais, como as redes de citações e cocitações (Otte \& Rousseau, 2002).

Os 133 artigos da amostra foram primeiramente distribuídos por ano; por autores; país de origem; periódicos; número de citações. Esta primeira etapa foi obtida através de tabelas e gráficos ilustrativos gerados pelo software Microsoft Excel ${ }^{\circledR}$. Ainda no que se refere a amostra de artigos, foi possível identificar: quais são os autores que mais a referenciam, o país e a instituição de pesquisa destes autores.

Na segunda etapa, elaborou-se as redes de citação e cocitação dos artigos da amostra e suas referências. Para tanto, foram utilizadas a ferramenta ResearcherlD ${ }^{\text {TM }}$ para relacionar os dados de saída do banco de dados WoS, e o programa VosViewer ${ }^{\circledR}$ para ilustrar as redes sociais dos artigos da amostra.

\section{ANÁLISE DOS RESULTADOS}

Uma primeira categoria de análise dos resultados (Figura 3) visa identificar tendências de crescimento ou decréscimo no interesse de desenvolvimento de estudos na área, classificando os artigos da amostra de acordo com o ano de publicação. Observou-se que a primeira publicação relacionada a flow shop permutacional envolvendo medidas de atraso data do ano de 1993. Deste ano até 2004 foram encontrados apenas 13 trabalhos publicados. A partir de 2005 nota-se maior constância na frequência, com destaque para o ano de 2011 e 2013, anos de maiores volumes de publicações.

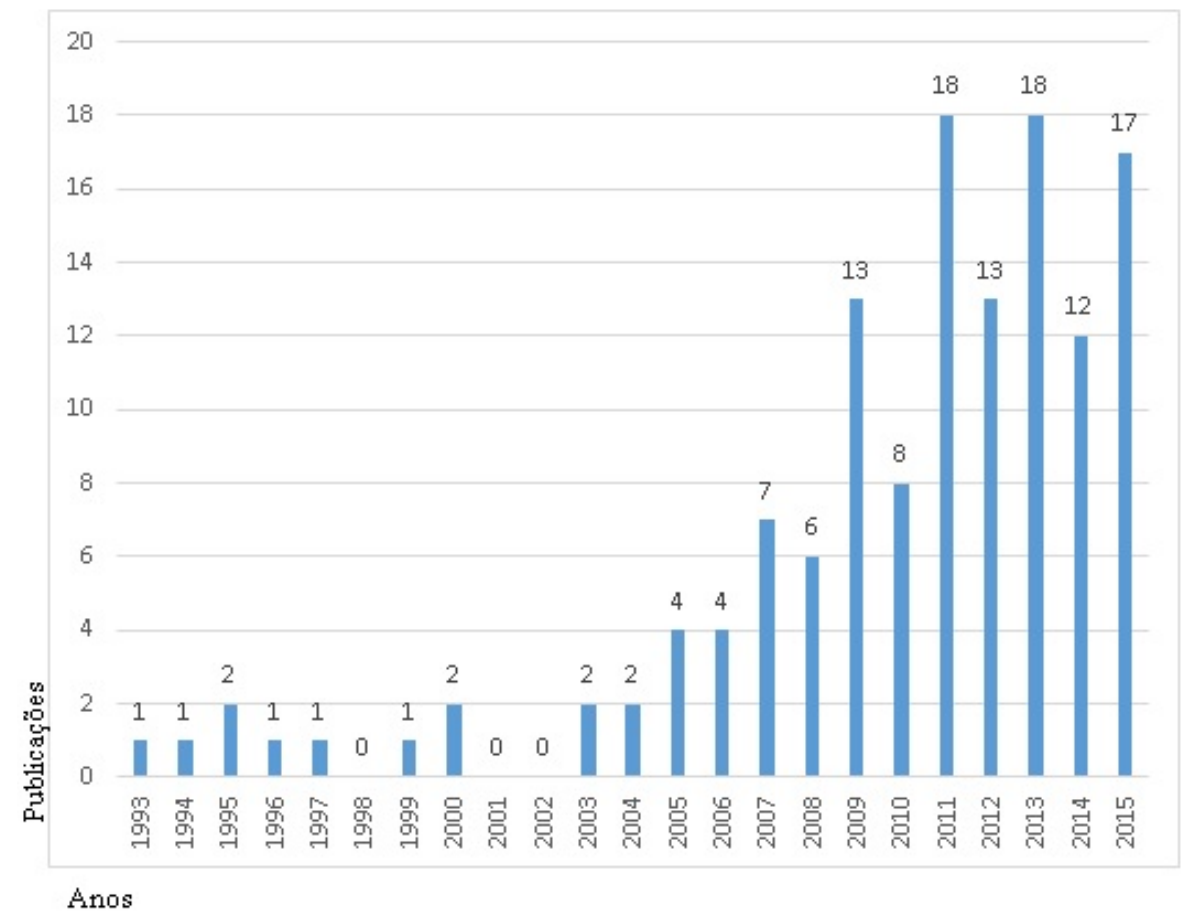

Figura 3: Evolução das publicações da amostra ao longo do tempo.

Fonte: Elaborada pelos autores 
Uma segunda análise descritiva procurou avaliar a dispersão dos trabalhos, a fim de identificar os autores, países de origem e periódicos em volume de publicações, conforme indicado na Figura 4, Figura 5 e Figura 6.

Com relação à distribuição dos trabalhos por autor (Figura 4), a análise da amostra revelou uma dispersão considerável. Durante o período analisado, 229 autores produziram pesquisas sobre o tema deste trabalho, sendo que os três mais produtivos foram responsáveis por 29 publicações.

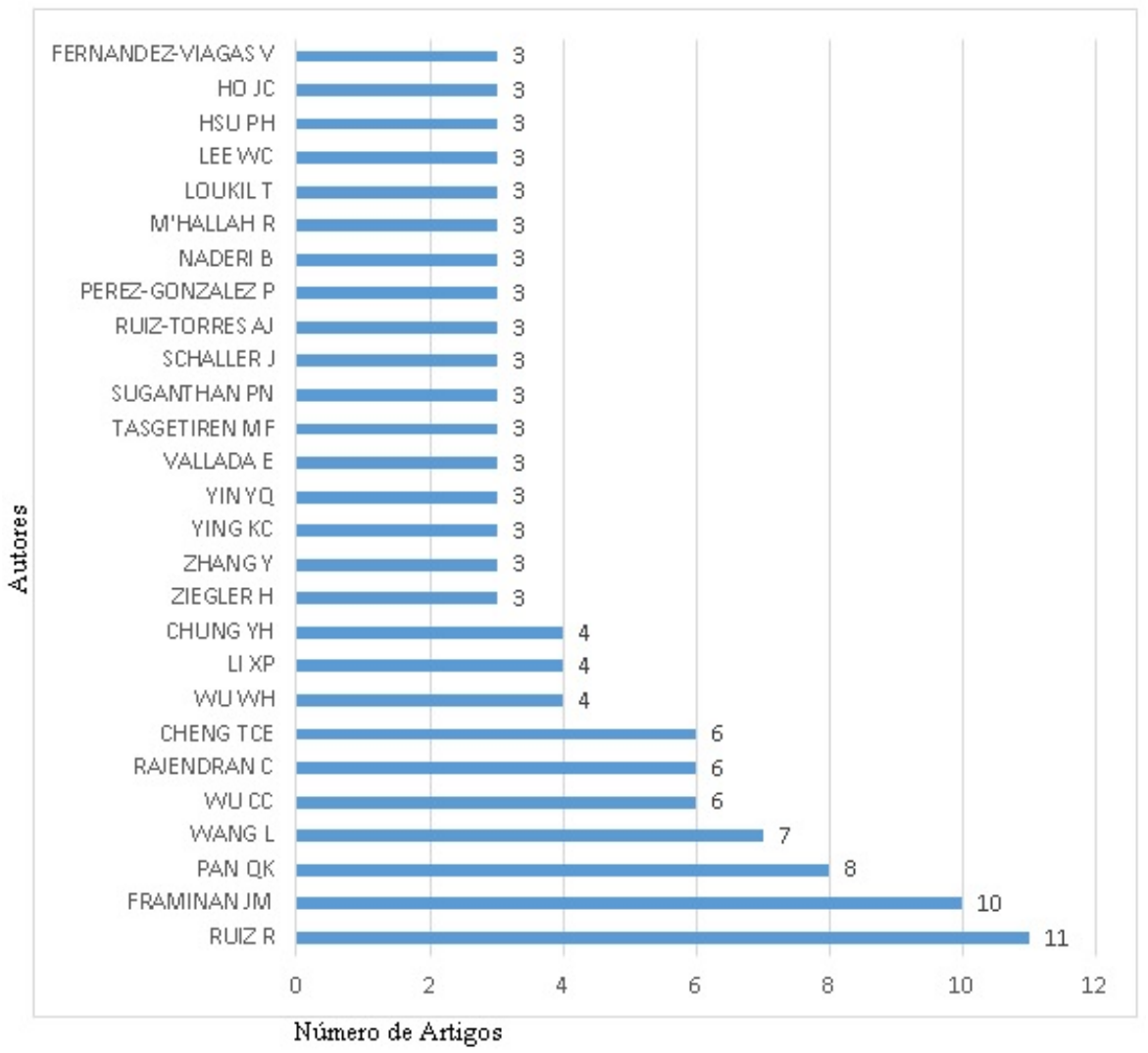

Figura 4: Autores com maior número de publicações da amostra.

Fonte: Elaborada pelos autores

A distribuição das publicações por país de origem demonstra um predomínio de publicações originárias da China, com publicação de 34 artigos, seguido dos Estados Unidos, Espanha e Taiwan, com publicação de 20 artigos cada. A Figura 5, estratifica todos os países de origem dos artigos da amostra.

As Universidades que despontam como centros de excelência em pesquisas sobre o tema são: a Universidade de Sevilha, na Espanha, seguida da Feng Chia University, de Taiwan e da Universidade Politécnica de Valência na Espanha. Outras escolas de destaque na publicação de artigos, são: Instituto de Tecnologia da Índia e as Universidades de Liaocheng e Tsinghua, ambas na China. 


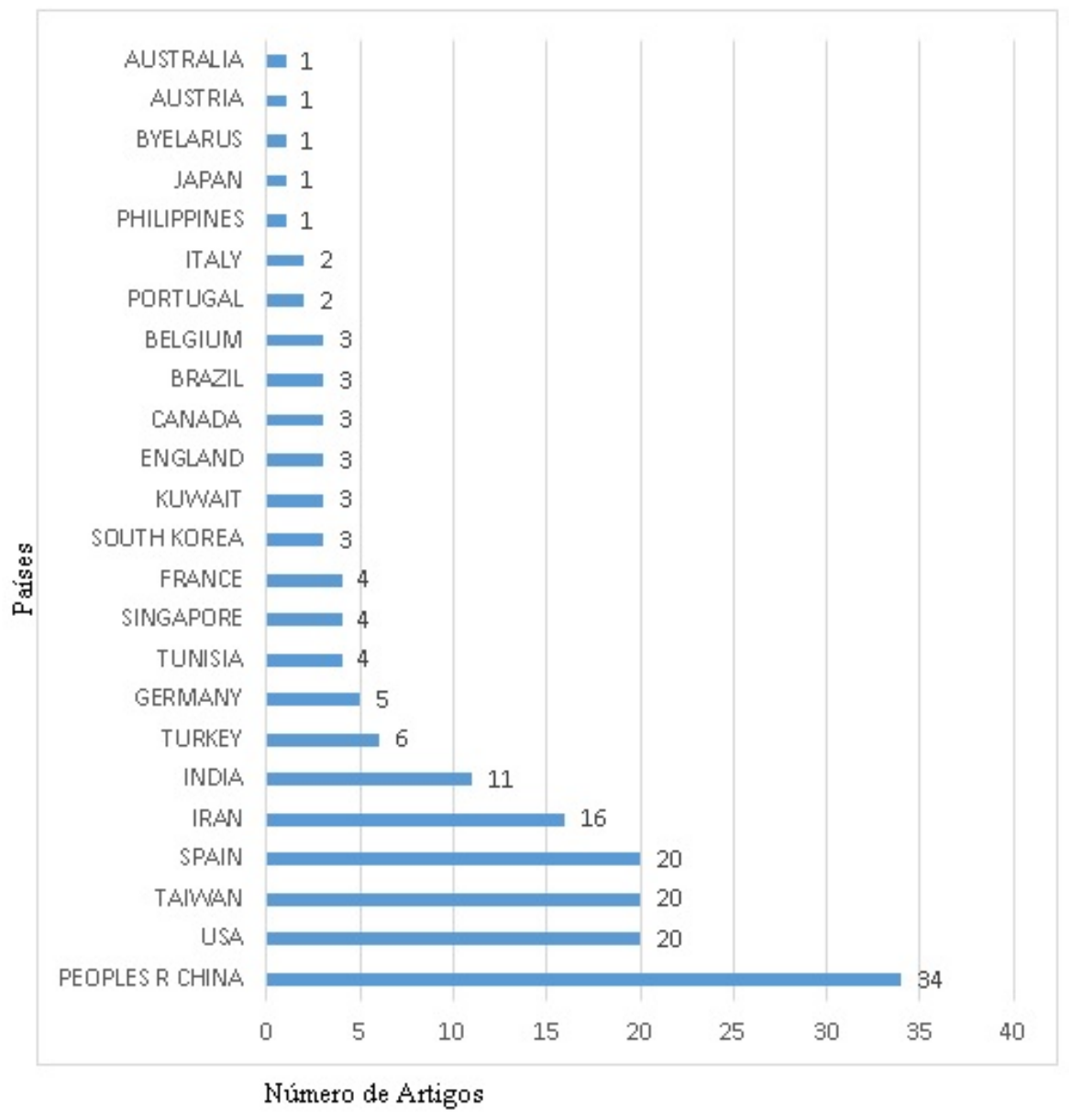

Figura 5: Distribuição das publicações da amostra por país de origem.

Fonte: Elaborada pelos autores

Com relação à distribuição dos artigos da amostra por revista de publicação (Figura 6), observou-se que, o periódico com maior destaque em volume de publicações é o International Journal of Production Research, seguido do Computers Operations Research (ambos do Reino Unido) e do European Journal of Operational Research da Holanda. Os 3 principais periódicos são responsáveis 51 publicações sobre o tema, o que representa pouco mais de $38 \%$ a amostra. 


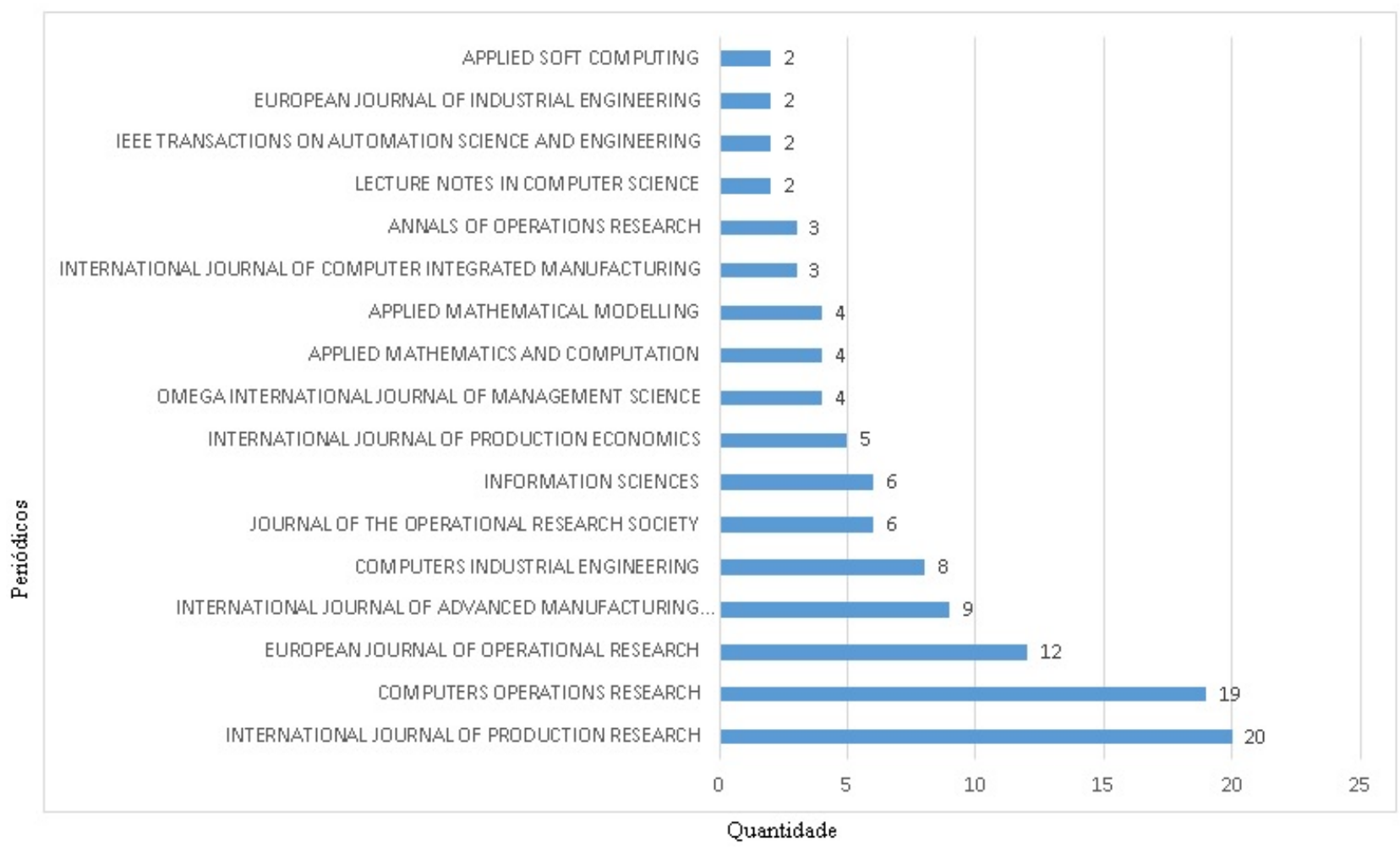

Figura 6: Distribuição das publicações da amostra pelos principais periódicos.

Fonte: Elaborada pelos autores

Os 133 artigos da amostra receberam 2449 citações, uma média de 106,48 citações ao ano e 18,41 por artigo. $\mathrm{O}$ índice $\mathrm{H}$ foi calculado em 25 , o que indica que a amostra possui 25 artigos com pelo menos 25 citações. Os artigos com os maiores números de citações da amostra são apresentados no Tabela 1. Também apresenta-se o fator de impacto de cada periódico, apurado pelo JCR, recurso que permite avaliar e comparar os periódicos e sua relevância em uma determinada área. $\mathrm{O}$ artigo de maior notoriedade pelo elevado número de citações é o de Rajendran e Ziegler (2004) com total um total de 221 citações (TC) e com média de citações por ano (MA) de 17,00. O artigo de Li e Ling (2007), publicado pela revista, IEEE Transactions on Systems, Man and Cybernetics Part B: Cybernetics, da Holanda possui maior fator de impacto (FI), dentre os 10 artigos mais citados.

Tabela 1: Publicações mais citadas da amostra.

\begin{tabular}{|c|c|c|c|c|}
\hline Autores (Ano da Publicação) & Título da Fonte & $\mathbf{F I}$ & TC & MA \\
\hline & EUROPEAN JOURNAL OF OPERATIONAL & & & \\
\hline Rajendran, C; Ziegler, H (2004) & RESEARCH & 2,679 & 221 & 17,00 \\
\hline $\begin{array}{l}\text { Pan, Quan-Ke; Tasgetiren, M. } \\
\text { Fatih; Suganthan, P. N.; Chua, T. J. } \\
\text { (2011) }\end{array}$ & INFORMATION SCIENCES & 3,364 & 174 & 29,00 \\
\hline $\begin{array}{l}\text { Yin, Yunqiang; Xu, Dehua; Sun, } \\
\text { Kaibiao; Li, Hongxing (2009) }\end{array}$ & INFORMATION SCIENCES & 3,364 & 111 & 13,88 \\
\hline Li, Bin-Bin; Wang, Ling (2007) & $\begin{array}{l}\text { IEEE TRANSACTIONS ON SYSTEMS MAN AND } \\
\text { CYBERNETICS PART B-CYBERNETICS }\end{array}$ & 6,220 & 103 & 10,30 \\
\hline $\begin{array}{l}\text { Pan, Quan-Ke; Wang, Ling; Qian, } \\
\text { Bin (2009) }\end{array}$ & COMPUTERS \& OPERATIONS RESEARCH & 1,988 & 69 & 8,62 \\
\hline
\end{tabular}




\begin{tabular}{llccc}
$\begin{array}{l}\text { Armentano, VA; Ronconi, DP } \\
\text { (1999) }\end{array}$ & COMPUTERS \& OPERATIONS RESEARCH & 1,988 & 65 & 3,61 \\
Zhang, Yi; Li, Xiaoping; Wang, & EUROPEAN JOURNAL OF OPERATIONAL & & \\
Qian (2009) & RESEARCH & 2,679 & 59 & 7,38 \\
KIM, YD (1993) & JOURNAL OF THE OPERATIONAL RESEARCH & & \\
SOCIETY & 1,225 & 59 & 2,46 \\
Vallada, Eva; Ruiz, Ruben (2010) & $\begin{array}{l}\text { OMEGA-INTERNATIONAL JOURNAL OF } \\
\text { MANAGEMENT SCIENCE }\end{array}$ & 3,962 & 58 & 8,29 \\
KIM, YD (1995) & EUROPEAN JOURNAL OF OPERATIONAL & 2,679 & 53 & 2,41 \\
\hline
\end{tabular}

A Figura 7 visa identificar tendências de crescimento ou decréscimo na quantidade de citações que a amostra recebeu, classificando-as por ano. Observa-se que a primeira citação ocorreu logo no ano subsequente à publicação mais antiga encontrada, Kim (1993). Nota-se que o período de aumento na frequência das citações está em consonância com a evolução das publicações apresentada na figura 3.

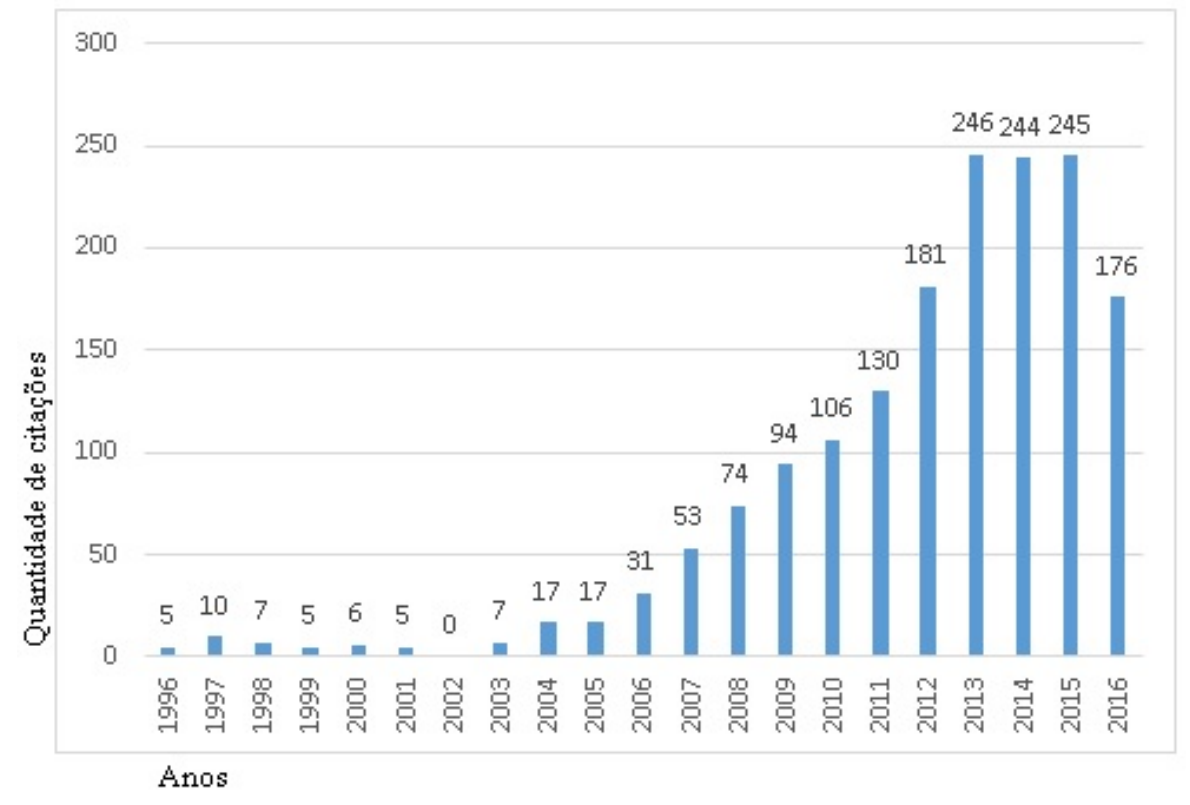

Figura 7: Evolução das citações dos artigos da amostra ao longo do tempo. Fonte: Elaborada pelos autores

Os autores que mais citaram as publicações da amostra foram ranqueados em ordem decrescente (Figura 8). Os vinte melhores colocados do ranking, foram responsáveis por cerca de $29 \%$ das citações ao longo do período analisado. Destaca-se que, os três autores que mais citaram a amostra foram responsáveis por 147 citações, o que representa quase $9 \%$ do total. 


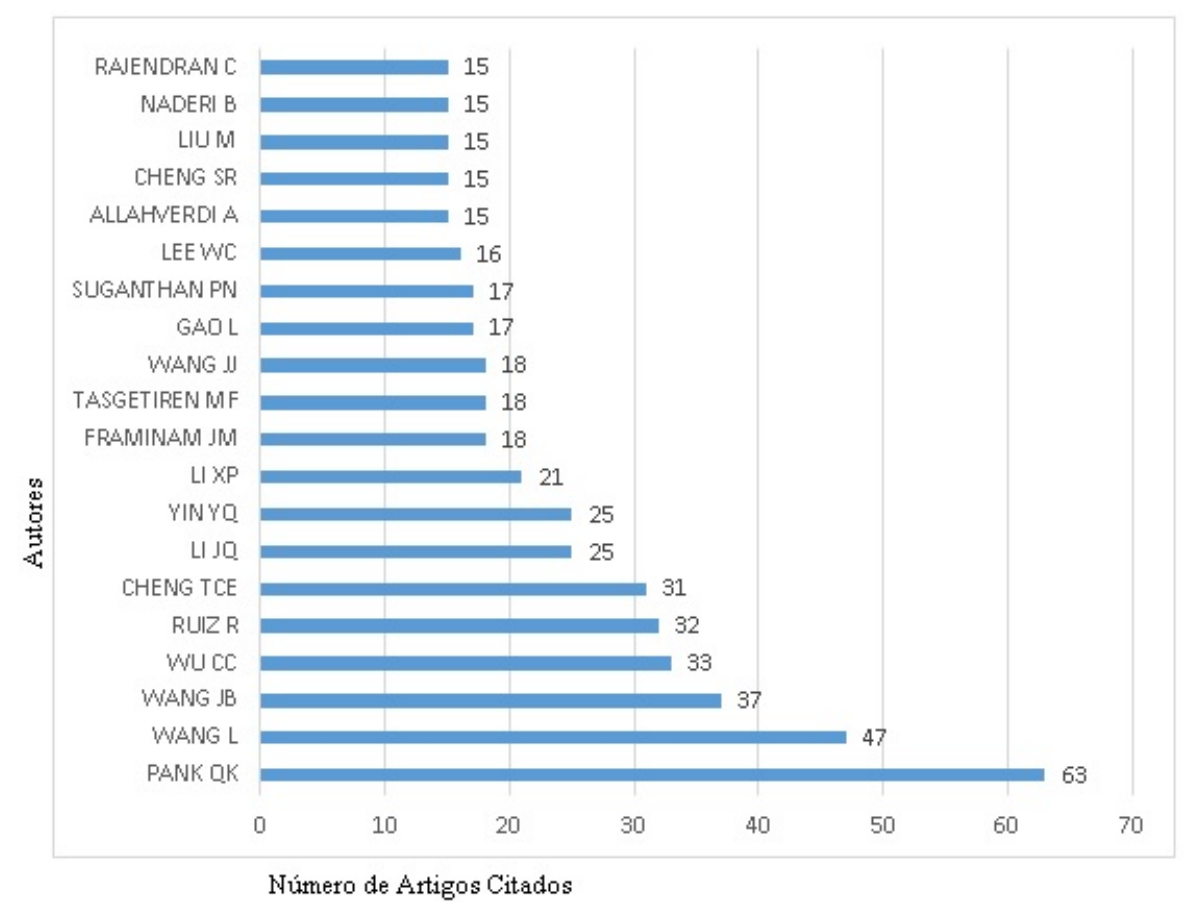

Figura 8: Autores que mais citaram as publicações da amostra.

Fonte: Elaborada pelos autores

A distribuição da origem das citações por país, demonstra um predomínio de ocorrências oriundas da China, com 670 citações. Os quatro países que mais citaram (China, Taiwan, Estados Unidos e Irã), representam pouco mais de $47 \%$ do total de citações que foram feitas envolvendo os artigos da amostra. A classificação com os 20 países que mais citaram os artigos da amostra é apresentada na Figura 9.

As universidades que despontam como centros que referenciam a amostra em suas pesquisas são: Tsinghua University e Liaocheng University, ambas chinesas. No Brasil, os artigos foram citados pela: Universidade Federal de Viçosa, Universidade Estadual de Campinas, Universidade Federal de Minas Gerais e Universidade de São Paulo. 


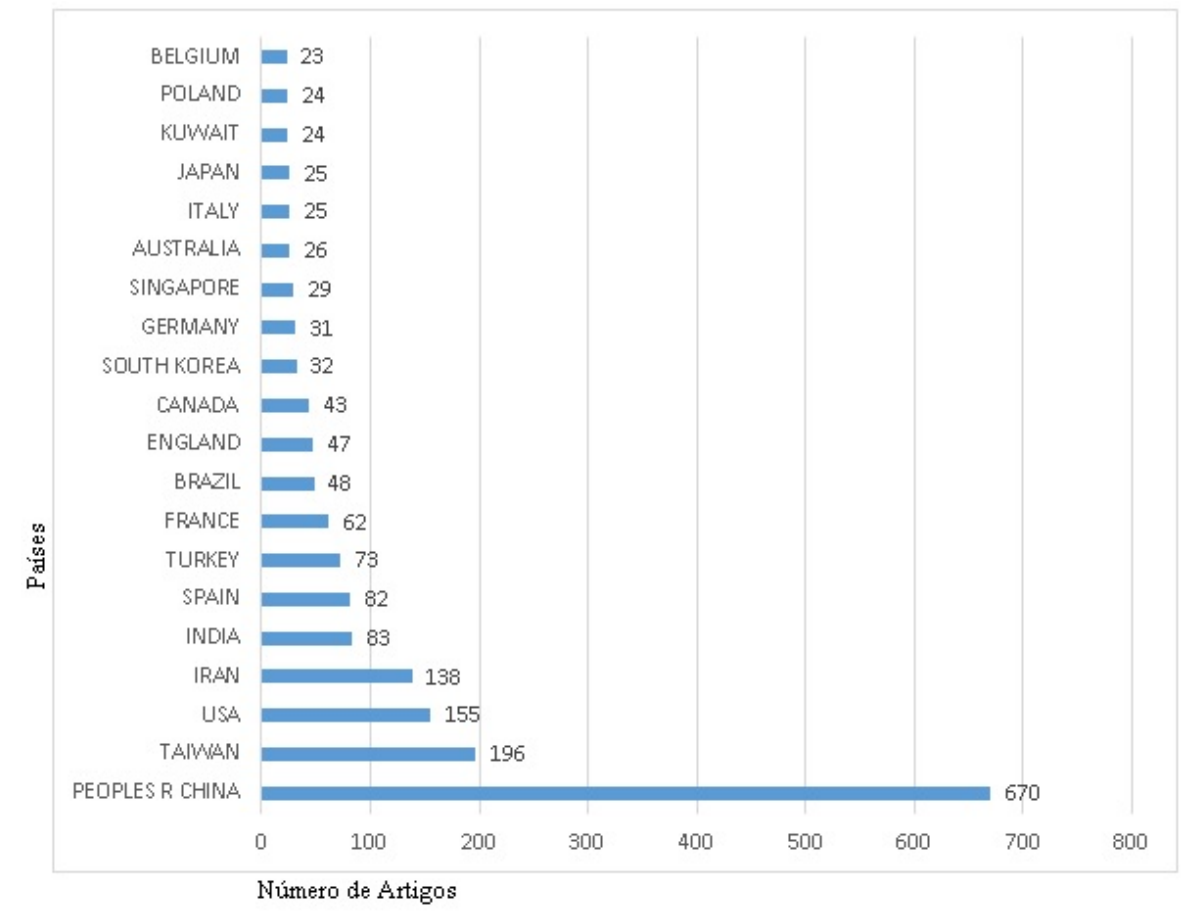

Figura 9: Distribuição das citações dos artigos por país.

Fonte: Elaborada pelos autores

$\mathrm{Na}$ busca de se demonstrar a interação entre os diferentes trabalhos, bem como a afinidade entre os pesquisadores, construiu-se uma rede social entre os artigos da amostra. A relação entre os elementos presentes é determinada com base no número de vezes que os autores citam uns aos outros. Os ícones representam os artigos da amostra que foram citados pelo menos 40 vezes. Seu tamanho é baseado no número de vezes em que cada artigo foi citado e suas cores indicam os grupos de nós muito conectados (coesos). O resultado dessa análise está demonstrado na Figura 10. A rede é altamente conectada, apresentando apenas 3 trabalhos em redes ou comunidades isoladas. Há uma concentração clara em torno da pesquisa de Armentano \& Ronconi (1999) que foi compartilhada por 4 artigos da rede.

daniels(1994)

li (2008) vallada (2010) armentano (1999) $\quad \operatorname{kim}(1995)$ $\operatorname{kim}(1993)$

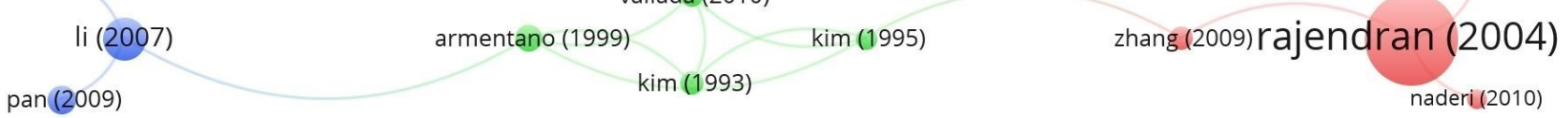


Como última etapa da análise de redes e com o objetivo de se medir o grau de ligação entre dois ou mais artigos pelo número de documentos onde esses artigos são citados simultaneamente, procedeu-se a análise de cocitação (Figura 11). Os ícones representam as referências dos artigos da amostra que foram citadas pelo menos 20 vezes, suas cores e tamanhos foram determinadas seguindo os mesmos critérios da rede de citações já apresentada. A pesquisa de Nawaz et al. (1983) foi a mais referenciada pela amostra. Destaca-se ainda, a presença de outros trabalhos clássicos e influentes de na área de scheduling como Johnson (1954), Taillard (1990,1993) e Pinedo (2002).

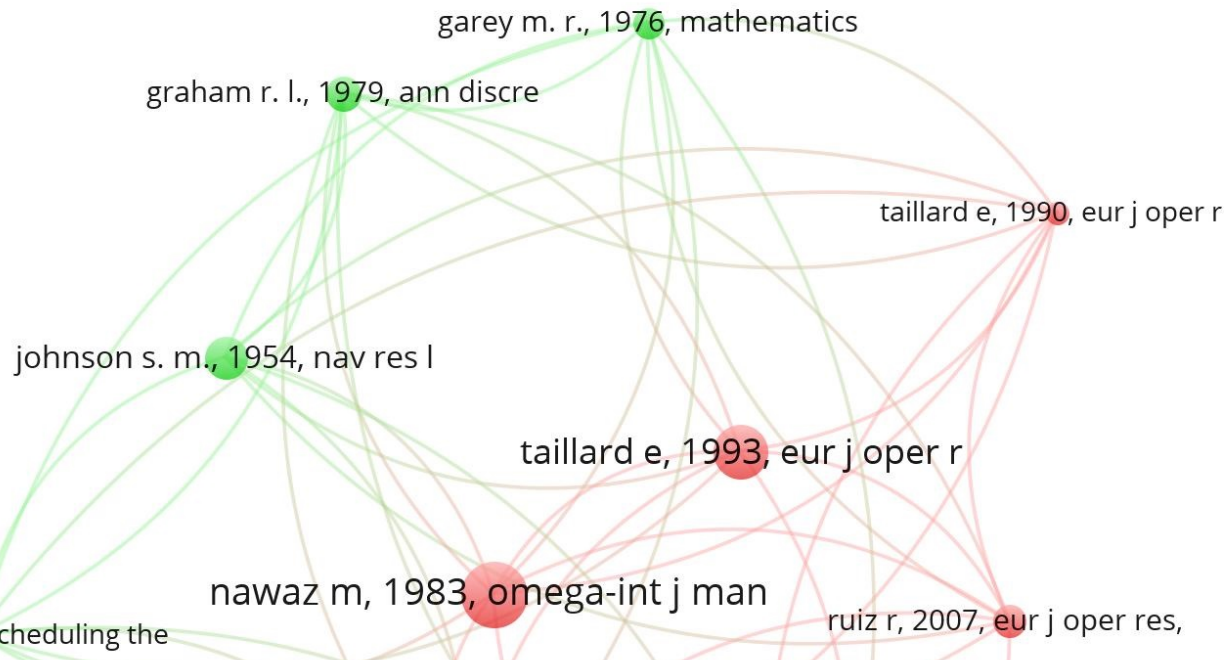

pinedo $\mathrm{m}, 2002$, scheduling the

nawaz m, 1983, omega-int j man

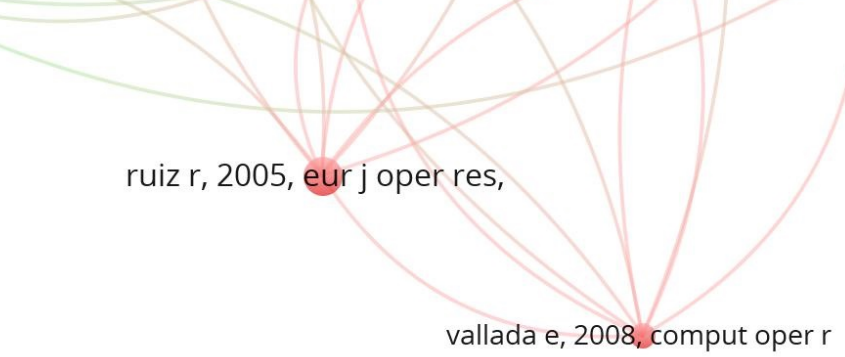

Figura 11: Rede de cocitações da amostra.

Fonte: Elaborada pelos autores

\section{CONSIDERAÇÕES FINAIS}

A identificação e organização das pesquisas que abordam programação da produção em flow shop permutacional envolvendo medidas de atraso contribui para a consolidação do conhecimento disponível e para o desenvolvimento de novas visões e conceitos. Significa também, um ponto de partida para pesquisadores encontrarem respostas que ainda não tenham sido dadas no referido campo de pesquisa.

A análise das publicações da base de dados Web of Science evidenciou 133 publicações relacionadas ao tema desta pesquisa. Constatou-se que a produção científica nesta área do conhecimento aumentou gradativamente ao longo dos últimos dez anos analisados, sendo a China o país de origem da maioria das publicações. A Universidade de Sevilha na Espanha desponta como centro de excelência em pesquisas relacionadas ao tema e o principal periódico 
em volume de publicações foi o International Journal of Production Research do Reino Unido. No que se refere à distribuição dos trabalhos por autor, verificou-se que o professor Rubén Ruiz da Universidade Politécnica de Valencia, foi responsável por 11 publicações.

Os artigos da amostra receberam 2449 citações, uma média de 106,48 citações ao ano e 18,41 por ano. Os trabalhos de maior notoriedade foram: Rajendran \& Ziegler (2004), pelo elevado número de citações e Li \& Ling (2007), pelo alto fator de impacto (FI) do periódico. Observou-se que o período de frequência das citações recebidas pela amostra acompanhou a evolução das publicações, evoluindo gradativamente ao longo dos últimos dez anos analisados. A distribuição da origem das citações por país demonstrou um predomínio de ocorrências oriundas da China. A Universidade de Tsighua, também na China, se destacou como centro que mais referenciou a amostra.

Em relação à rede de citações, verificou-se uma rede altamente conectada, com apenas 3 trabalhos em redes ou comunidades isoladas e uma concentração em torno do trabalho de Armentano \& Ronconi (1999). A rede de cocitações por sua vez demonstrou que a pesquisa de Nawaz et al. (1983) foi a mais referenciada pela amostra.

Houve algumas limitações na pesquisa, uma delas refere-se ao viés da base de dados escolhida. É possível que existam resultados de pesquisa bastante significativos que, por não estarem disponíveis em inglês ou na WoS, não fizeram parte da amostra e consequentemente não foram retratadas nesta análise.

Outra limitação: a análise do conhecimento foi feita por meio das publicações e por este motivo, evidentemente não foi possível mensurar os conhecimentos empíricos ou que não tenham sido reportados em artigos científicos.

Dentre as várias lacunas de pesquisa encontradas, identificamos um importante aspecto a ser explorado em pesquisas futuras: a investigação bibliométrica de publicações com flow shop não permutacional e outros ambientes clássicos, como máquinas paralelas e job shop, por exemplo, e focando outras medidas além do atraso, como fluxos, custos e esperas.

\section{AGRADECIMENTOS}

Agradecemos às agências de fomento FAPEG e CNPq pelo apoio e auxílio financeiro, primordiais para a realização do presente estudo e aos revisores anônimos pela leitura cuidadosa do texto.

\section{REFERÊNCIAS}

Alves, A. D., Yanasse, H. H., \& Soma, N. Y. (2016). An analysis of bibliometric indicators to JCR according to Benford's law. Scientometrics, 107(3), 1489-1499.

Armentano, V. A., \& Ronconi, D. P. (1999). Tabu search for total tardiness minimization in flowshop scheduling problems. Computers \& operations research, 26(3), 219-235.

Baker, K. R. (1974). Introduction to sequencing and scheduling. John Wiley \& Sons. 
Campbell, H. G., Dudek, R. A., \& Smith, M. L. (1970). A heuristic algorithm for the $\mathrm{n}$ job, $\mathrm{m}$ machine sequencing problem. Management science, 16(10), B-630.

Carvalho, M., Fleury, A., \& Lopes, A. P. (2013). An overview of the literature on technology roadmapping (TRM): Contributions and trends. Technological Forecasting and Social Change, 80(7), 1418-1437.

Endler, K. D., Steiner, M. T. A., Scarpin, C. T., \& de Souza, P. L. B. (2015). Operations Programming in Production Systems Permutation Flow Shop: A Bibliometric Contribution. IEEE Latin America Transactions, 13(12), 3966-3973.

Fernandez-Viagas, V., \& Framinan, J. M. (2015). NEH-based heuristics for the permutation flowshop scheduling problem to minimise total tardiness. Computers \& Operations Research, 60, 27-36.

Framinan, J. M., \& Leisten, R. (2008). Total tardiness minimization in permutation flow shops: a simple approach based on a variable greedy algorithm. International Journal of Production Research, 46(22), 6479-6498.

Fuchigami, H. Y. (2015). Sequenciamento da produção em sistemas flow shop. UFG, Goiânia-GO.

Fuchigami, H. Y., \& Rangel, S. (2015). Métodos heurísticos para maximização do número de tarefas just-in-time em flow shop permutacional. XLVII Simpósio Brasileiro de Pesquisa Operacional, Porto de Galinhas-PE.

Graham, R. L., Lawler, E. L., Lenstra, J. K., \& Kan, A. R. (1979). Optimization and approximation in deterministic sequencing and scheduling: a survey. Annals of discrete mathematics, 5, 287326.

Gumpenberger, C., Wieland, M., \& Gorraiz, J. (2012). Bibliometric practices and activities at the University of Vienna. Library Management, 33(3), 174-183.

Gupta, J. N. (1971). A functional heuristic algorithm for the flowshop scheduling problem. Journal of the Operational Research Society, 22(1), 39-47.

Johnson, S. M. (1954). Optimal two-and three-stage production schedules with setup times included. Naval Research Logistics (NRL), 1(1), 61-68.

Hundal, T. S., \& Rajgopal, J. (1988). An extension of Palmer's heuristic for the flow shop scheduling problem. International Journal of Production Research, 26(6), 1119-1124.

Kim, Y. D. (1993). Heuristics for flowshop scheduling problems minimizing mean tardiness. Journal of the Operational Research Society, 19-28.

Kim, Y. D. (1995). Minimizing total tardiness in permutation flowshops. European Journal of Operational Research, 85(3), 541-555. 
Lee, W. C., Yeh, W. C., \& Chung, Y. H. (2014). Total tardiness minimization in permutation flowshop with deterioration consideration. Applied Mathematical Modelling, 38(13), 30813092.

Li, B. B., \& Wang, L. (2007). A hybrid quantum-inspired genetic algorithm for multiobjective flow shop scheduling. IEEE Transactions on Systems, Man, and Cybernetics, Part B (Cybernetics), 37(3), 576-591.

Lopez, P., \& Roubellat, F. (2013). Production scheduling. John Wiley \& Sons.

M'Hallah, R. (2014). Minimizing total earliness and tardiness on a permutation flow shop using VNS and MIP. Computers \& Industrial Engineering, 75, 142-156.

Maccarthy, B. L., \& Liu, J. (1993). Addressing the gap in scheduling research: a review of optimization and heuristic methods in production scheduling. The International Journal of Production Research, 31(1), 59-79.

Madhushini, N., Rajendran, C., \& Deepa, Y. (2009). Branch-and-bound algorithms for scheduling in permutation flowshops to minimize the sum of weighted flowtime/sum of weighted tardiness/sum of weighted flowtime and weighted tardiness/sum of weighted flowtime, weighted tardiness and weighted earliness of jobs. Journal of the Operational Research Society, 60(7), 991-1004.

Moccellin, J. V. (1999). Programação da produção em sistemas Flowshop utilizando um método heurístico híbrido algoritmo genético-busca tabu. Encontro Nacional de Engenharia de Produção, Rio de Janeiro - RJ.

Moccellin, J. V., \& Nagano, M. S. (2007). Uma propriedade estrutural do problema de programação da produção flow shop permutacional com tempos de setup. Pesquisa Operacional, 27(3), 487-515.

Nawaz, M., Enscore, E. E., \& Ham, I. (1983). A heuristic algorithm for the m-machine, n-job flowshop sequencing problem. Omega, 11(1), 91-95.

Osman, I. H., \& Laporte, G. (1996). Metaheuristics: A bibliography.

Otte, E., \& Rousseau, R. (2002). Social network analysis: a powerful strategy, also for the information sciences. Journal of information Science, 28(6), 441-453.

Packer, A. L., \& Meneghini, R. (2006). Visibilidade da produção científica. Comunicação \& produção científica: contexto, indicadores e avaliação. São Paulo: Angellara, 237-259.

Palmer, D. S. (1965). Sequencing jobs through a multi-stage process in the minimum total timea quick method of obtaining a near optimum. Journal of the Operational Research Society, 16(1), 101-107. 
Pilkington, A., \& Meredith, J. (2009). The evolution of the intellectual structure of operations management-1980-2006: A citation/co-citation analysis. Journal of Operations Management, 27(3), 185-202.

Pinedo, M. L. (2002). Scheduling: theory, algorithms, and systems. Springer.

Pinedo, M. L. (2012). Scheduling: theory, algorithms, and systems. Springer.

Rajendran, C., \& Ziegler, H. (2004). Ant-colony algorithms for permutation flowshop scheduling to minimize makespan/total flowtime of jobs. European Journal of Operational Research, 155(2), 426-438.

Schaller, J. (2012). Scheduling a permutation flow shop with family setups to minimise total tardiness. International Journal of Production Research, 50(8), 2204-2217.

Schaller, J., \& Valente, J. M. (2013). A comparison of metaheuristic procedures to schedule jobs in a permutation flow shop to minimise total earliness and tardiness. International Journal of Production Research, 51(3), 772-779.

Silva, M. R. D. (2004). Análise biliométrica da produção científica docente do Programa de PósGraduação em Educação Especial da UFSCar: 1998-2003.

Taillard, E. (1990). Some efficient heuristic methods for the flow shop sequencing problem. European journal of Operational research, 47(1), 65-74.

Taillard, E. (1993). Benchmarks for basic scheduling problems. european journal of operational research, 64(2), 278-285.

Tasgetiren, M. F., Pan, Q. K., Suganthan, P. N., \& Oner, A. (2013). A discrete artificial bee colony algorithm for the no-idle permutation flowshop scheduling problem with the total tardiness criterion. Applied Mathematical Modelling, 37(10), 6758-6779.

Testa, J. (2011). The globalization of Web of Science: 2005-2010. Thomson Reuters. Philapelphia, $P A$.

Vallada, E., \& Ruiz, R. (2010). Genetic algorithms with path relinking for the minimum tardiness permutation flowshop problem. Omega, 38(1), 57-67.

WOS, Web of Science. Disponível em: http://ipscience.thomsonreuters.com/product/web-ofscience/. Acesso em: 21 abr. 2016.

Xiao, Y. Y., Zhang, R. Q., Zhao, Q. H., \& Kaku, I. (2012). Permutation flow shop scheduling with order acceptance and weighted tardiness. Applied Mathematics and Computation, 218(15), 7911-7926. 